\title{
An analytical approach to initiation of propagating fronts
}

\author{
I. Idris and V. N. Biktashev \\ Department of Mathematical Sciences, University of Liverpool, Liverpool L69 7ZL, UK
}

(Dated: October 31, 2018)

\begin{abstract}
We consider the problem of initiation of a propagating wave in a one-dimensional excitable fibre. In the Zeldovich-Frank-Kamenetsky equation, a.k.a. Nagumo equation, the key role is played by the "critical nucleus" solution whose stable manifold is the threshold surface separating initial conditions leading to initiation of propagation and to decay. Approximation of this manifold by its tangent linear space yields an analytical criterion of initiation which is in a good agreement with direct numerical simulations.

PACS numbers: 87.10.+e, 02.90.+p
\end{abstract}

Threshold phenomena are widespread in bistable dissipative systems. If such a system is spatially extended, then fronts switching from one local state to the other can propagate. Propagating fronts, or trigger waves, play important roles in such diverse physical situations as selfheating in metals and superconductors, phase transitions, combustion and other chemical reaction waves, and biological signalling systems [1-6] to name a few. In biology and chemistry they often appear as a fast stage of pulse waves in "excitable systems" [3,6-8]. The question of existence of such waves in particular mathematical models is well studied. However, whether a propagating wave will actually be observed depends on initial conditions. Understanding conditions of initiation of propagating fronts or pulses is very important in applications. In heart such waves trigger coordinated contraction of the muscle and failure of initiation can cause or contribute to serious or fatal medical conditions, or render inefficient the work of pacemakers or defibrillators [9]. In combustion, understanding of initiation is of critical importance for safety in storage and transport of combustible materials [10].

Mathematically, after the external initiating stimulus has finished, the problem is reduced to classification of initial conditions that will or will not lead to a propagating wave solution. This problem is difficult as it is essentially non-stationary, spatially extended and nonlinear, and does not have any helpful symmetries. Yet the problem is so important that analytical answers are highly desirable even if not very accurate.

Early attempts of analytical treatment of the initiation problems, including the spatially extended ones, used linear description supplemented with heuristic conditions to represent the threshold [11-15] and, more recently, lowdimensional Galerkin style approximations of the partial differential equations $[16,17]$

In the last two decades, this problem has been analysed from the dynamical systems theory viewpoint [1622]. These studies identified the importance of certain "critical solutions", whose codimension-1 (center-)stable manifold acts as the critical surface separating the basins of attraction of initiation and decay. This understanding was used in sophisticated numerical methods of calculating initiation thresholds, e.g. [21].

Here we propose a practical method of defining the initiation criteria analytically. The idea is based on the linearization of the (center-)stable manifold of the critical solution by its linear tangent, the (center-)stable space. One would expect that this should work well for initial conditions sufficiently close to the critical nucleus. However, how close it should be to give a reasonable approximation is not clear a priori. We consider a test case with very crude initial conditions, in the form of rectangular pulses, and the analytical criterion gives surprisingly good agreement with direct numerical simulations.

We consider a one-component reaction-diffusion equation

$$
u_{t}=u_{x x}+f(u),
$$

with bistable kinetics $f(u)$. As an archetypical example, we consider Zeldovich-Frank-Kamenetsky equation suggested to describe flame propagation [23], which is also known as Nagumo equation in its capacity as the fast equation in the FitzHugh-Nagumo system, suggested as a simplified model of nerve conduction [24, 25]. This equation has the kinetics in the form

$$
f(u)=u(u-\theta)(1-u), \quad \theta=\text { const }<1 / 2 .
$$

Equations (1) have propagating front solutions,

$$
u=U(x-c t-\Delta), \quad \Delta=\text { const }
$$

e.g. for (2),

$$
U(\xi)=\frac{1}{1+e^{\xi / \sqrt{2}}}, \quad c=\frac{1-2 \theta}{\sqrt{2}} .
$$

We consider a half-infinite cable which is driven away at $t=0$ from the resting state $u=0$ by an instantaneous stimulus of amplitude $u_{s}$ and spatial extent $x_{s}$ at $t=0$ and/or by a current injection at $x=0$ of amplitude $I_{s}$ lasting for time $t_{s}$,

$$
\begin{aligned}
& u_{t}=u_{x x}+f(u), \quad(x, t) \in \mathbb{R}^{+} \times \mathbb{R}^{+}, \\
& u_{x}(0, t)=I_{s} g\left(t ; t_{s}\right), \quad g\left(+\infty ; t_{s}\right)=0, \\
& u(x, 0)=u_{s} h\left(x ; x_{s}\right), \quad h\left(+\infty ; x_{s}\right)=0,
\end{aligned}
$$




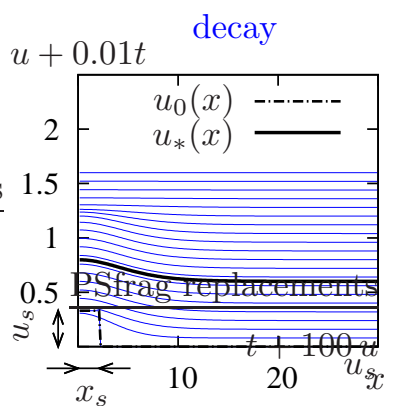

(a)

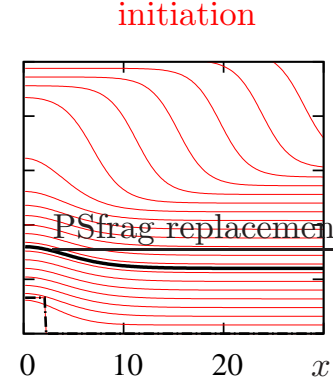

(b)

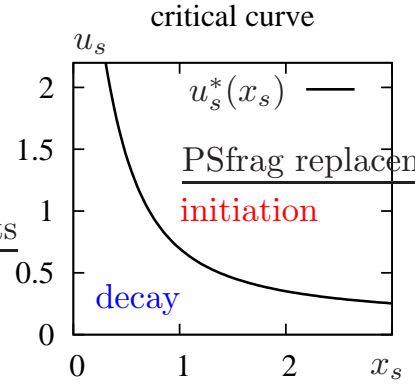

(c)

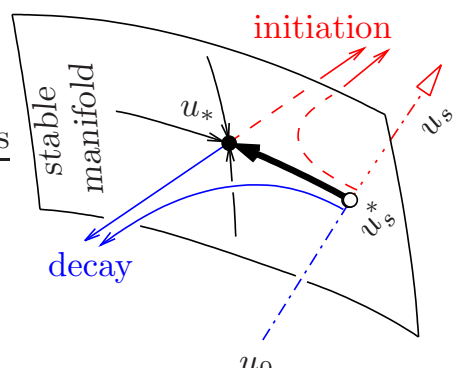

(d)

FIG. 1: (color online) (a,b) Response to an below- and above-threshold initial perturbation in ZFK equation, (1,2,8). Parameter values: $\theta=0.13, I_{s}=0, x_{s}=2.10$ for both, subthreshold $u_{s}=0.3304831$ (a) and superthreshold $u_{s}=0.3304833$ (b) cases, numerics using central difference centered in space with step $h_{x}=0.15$ and forward Euler in time with step $h_{t}=0.01$. Dashdotted black lines: initial conditions, bold solid black lines: the critical nuclei. (c) The corresponding critical strength-extent curve, separating initiation initial conditions from decay initial conditions. (d) The sketch of a stable manifold of the critical solution for the ZFK equation. The critical nucleus is represented by the black dot; the critical trajectories, constituting the stable manifold, are shown in black. The family of initial conditions is represented by the dash-dotted line. The bold black line is the critical trajectory with initial condition in that family. The sub-threshold trajectories are represented by the blue line, while the red lines represent super-threshold trajectories. Note that the point where the initial condition intersect the stable manifold is shown as the empty circle.

or equivalently

$$
\begin{aligned}
& u_{t}=u_{x x}+f(u)+2 I_{s} g\left(t ; t_{s}\right) \delta(x), \\
& (x, t) \in \mathbb{R} \times \mathbb{R}^{+} ; \\
& u(x, 0)= \begin{cases}u_{s} h\left(x ; x_{s}\right), & x \geq 0, \\
u_{s} h\left(-x ; x_{s}\right), & x<0 .\end{cases}
\end{aligned}
$$

where $\delta()$ is the Dirac delta function (generalization for a generic stimulus $I_{s} g(x, t)$ is straighforward). Specifically, we consider stimuli of rectangular profiles,

$$
g\left(t ; t_{s}\right)=\Theta\left(t_{s}-t\right), \quad h\left(x ; x_{s}\right)=\Theta\left(x_{s}-x\right),
$$

where $\Theta()$ is the Heaviside step function.

Depending on parameters $u_{s}, x_{s}, I_{s}$ and $t_{s}$, problem $(3,4,5)$ can typically produce either a "decay" solution such that $\max _{x} u(x, t) \rightarrow 0, t \rightarrow \infty$, see Fig. 1(a), or an "initiation" solution such that $\max _{x} \mid u(x, t)-U(x-c t-$ $\Delta) \mid \rightarrow 0, t \rightarrow \infty$ for some $\Delta \in \mathbb{R}$, see Fig. $1(\mathrm{~b})$. Naturally, in the even extension $(6,7)$, the "initiation" solution produces two fronts propagating both ways. Our goal is a condition that would predict which of the two outcomes will take place for given $u_{s}, x_{s}, I_{s}$ and $t_{s}$. The curve in the $\left(t_{s}, I_{s}\right)$ plane, at $u_{s}=0$, separating the two outcomes, is widely known as the strength-duration curve. We will also consider a similar critical curve in the $\left(x_{s}, u_{s}\right)$ plane at $I_{s}=0$, see Fig. 1(c), which we will call strength-extent curve.

We consider first the case $I_{s}=0$, and following [19], review the fundamental role of the critical nucleus solution $u_{*}(x)$, which is defined as a nontrivial stationary solution of (1), i.e.

$$
u_{*}^{\prime \prime}+f\left(u_{*}\right)=0, \quad u_{*}(x) \neq \text { const },
$$

e.g. for (2),

$$
u_{*}(x)=\frac{3 \theta \sqrt{2}}{(1+\theta) \sqrt{2}+\cosh (x \sqrt{2}) \sqrt{2-5 \theta+\theta^{2}}} .
$$

It is then demonstrated that such a solution is unstable. Consider linearization of (1) near it, $u(x, t)=u_{*}(x)+$ $v(x, t), \quad v(x, t) \ll 1$, then $v_{t}=\mathcal{L} v$, where $\mathcal{L}=\partial_{x}^{2}+$ $f_{u}\left(u_{*}(x)\right)$. Stability of $u_{*}$ is determined by the spectrum of $\mathcal{L}$,

$$
\mathcal{L} \phi_{j}=\lambda_{j} \phi_{j}
$$

Since $\mathcal{L}$ is a Sturm-Liouville operator, all its eigenvalues $\lambda_{j}$ are real.

Notice that $\mathcal{L} \partial_{x} u_{*}=0$ so $u_{*}^{\prime}(x)$ is an eigenfunction corresponding to eigenvalue 0. By Sturm's oscillation theorem, if eigenvalues of the discrete spectrum are ordered so that $\lambda_{1}>\lambda_{2}>\lambda_{3}>\ldots$ then egenfunction $\phi_{j}$ shall have precisely $j-1$ zeros. The critical nucleus $u_{*}(x)$ is an even function and has a single maximum at $x=0$, so $u_{*}^{\prime}(x)$ has exactly one zero, and therefore we have

$$
C_{2} u_{*}^{\prime}(x)=\phi_{2}, \quad \lambda_{2}=0,
$$

for some $C_{2} \neq 0$. This implies that $u_{*}$ is unstable, and there is exactly one positive eigenvalue, $\lambda_{1}>0$, with a corresponding $\phi_{1}(x)>0$. The continuous spectrum of $\mathcal{L}$ is $\{\lambda\}=\left(-\infty, \lambda_{c}\right]$, where $\lambda_{c}=\lim _{x \rightarrow \pm \infty}\left[\partial_{u} f(u)\right]_{u=u_{*}(x)}=$ $f^{\prime}(0)<0$. Hence in the phase space of $(6)$, equilibrium $u_{*}$ is a saddle point, with only one unstable direction. Its stable manifold [27] has therefore codimension one and, as such, partitions the phase space. One part of the phase space corresponds to the decay solutions, and the other to the initiation solutions (Fig. 1(d)). A oneparametric family of initial conditions (5), say with a 
fixed $x_{s}>0$ and the parameter $u_{s}$, will cross the stable manifold once, say at $u_{s}=u_{s}^{*}\left(x_{s}\right)$. For $u_{s}<u_{s}^{*}\left(x_{s}\right)$, we have decay, and for $u_{s}>u_{s}^{*}\left(x_{s}\right)$ initiation. This defines the strength-extent curve $u_{s}=u_{s}^{*}\left(x_{s}\right)$. The role of the stable manifold of the critical nucleus $u_{*}$ as the threshold surface in the phase space is an empirically verifiable fact: it means that the critical nucleus will be observed as a transient for any initial conditions sufficiently close to the threshold (see Fig. 1 (a,b) for $t \lesssim 100$ ).

Now we shall use this understanding to construct an analytical criterion of initiation. Our idea is to replace the stable manifold of $u_{*}$ by its tangent, i.e. the stable space. This implies considering the initiation problem in the linear approximation around $u_{*}(x)$. Continuing with the case $I_{s}=0$, we get

$$
u(x, t)=u_{*}(x)+\sum_{j=1}^{\infty} a_{j} e^{\lambda_{j} t} \phi_{j}(x),
$$

where for brevity the summation is assumed both over the discrete and the continuous spectrum. If we choose the eigenfunctions $\phi_{j}(x)$ normalized, then $a_{j}=$ $\int_{-\infty}^{\infty} \phi_{j}(x)\left(u(x, 0)-u_{*}(x)\right) \mathrm{d} x$. Eigenfunction $\phi_{2}(x)=$ $u_{*}^{\prime}(x)$ is odd, $u_{*}(x)$ and $u(x, 0)$ are even, hence $a_{2}=0$, and $\sum_{j=3}^{\infty} a_{j} e^{\lambda_{j} t} \phi_{j}(x) \rightarrow 0$ as $t \rightarrow \infty$ since $\lambda_{j} \leq \lambda_{3}<0$ for $j \geq 3$. Hence in this approximation $u(x, t) \rightarrow u_{*}(x)$ if and only if $a_{1}=0$. So the equation of the stable space, which is an approximation of the critical manifold, is $a_{1}=0$ or

$$
\int_{0}^{\infty} \phi_{1}(x)\left(u_{s} h\left(x ; x_{s}\right)-u_{*}(x)\right) \mathrm{d} x=0 .
$$

This is a finite equation for $x_{s}, u_{s}$, which provides the desired analytical definition of the strength-extent curve.

For $I_{s} \neq 0$, we have

$$
u(x, t)=u_{*}(x)+\sum_{j=1}^{\infty} A_{j}(t) \phi_{j}(x),
$$

where $A_{j}(0)=\int_{-\infty}^{\infty} \phi_{j}(x)\left(u(x, 0)-u_{*}(x)\right) \mathrm{d} x$ and $\mathrm{d} A_{j} / \mathrm{d} t=\lambda_{j} A_{j}+2 I_{s} g(t) \phi_{j}(0)$, which can be solved in quadratures for a given $g(t)$, and then the critical condition is $A_{1}(+\infty)=0$, or

$$
A_{1}(0)+2 I_{s} \phi_{1}(0) \int_{0}^{\infty} e^{-\lambda_{1} t} g(t) \mathrm{d} t=0 .
$$

Now we consider an example with explicit answers. For (2), if $\theta \ll 1$, then $u_{*}=\varnothing \theta$, and as in [17], for $u \lesssim \theta$ we can approximate

$$
f(u) \approx u(u-\theta)
$$

and then $u_{*} \approx \frac{3}{2} \theta \operatorname{sech}^{2}(x \sqrt{\theta} / 2)$. In this approximation, to solve the eigenvalue problem (9), it is convenient to change variables $v(x)=\psi(z), z=\tanh (x \sqrt{\theta} / 2)$, then

$$
\left(\left(1-z^{2}\right) \psi^{\prime}\right)^{\prime}+\left(12-\frac{4(1+\lambda / \theta)}{1-z^{2}}\right) \psi=0, \quad \psi( \pm 1)=0,
$$

solutions of which are associated Legendre functions [26]. In particular, we find that

$$
\lambda_{1}=5 \theta / 4, \quad \phi_{1}(x)=C_{1} \operatorname{sech}^{3}(x \sqrt{\theta} / 2)
$$

for some $C_{1} \neq 0$.

For $I_{s}=0$ and $h\left(x ; x_{s}\right)=\Theta\left(x_{s}-x\right)$, equation (10) then gives an explicit equation for the strength-extent curve

$$
\begin{array}{r}
u_{s}=\frac{9 \theta}{8}\left[\frac{2}{\pi} \tanh \left(\frac{x_{s} \sqrt{\theta}}{2}\right) \operatorname{sech}\left(\frac{x_{s} \sqrt{\theta}}{2}\right)\right. \\
\left.+\frac{4}{\pi} \arctan \left(e^{x_{s} \sqrt{\theta} / 2}\right)-1\right]^{-1} .
\end{array}
$$

For $u_{s}=0$ and $g\left(t ; t_{s}\right)=\Theta\left(t_{s}-t\right)$, we have $A_{1}(0)=\frac{9}{8} \pi \sqrt{\theta} C_{1}$ and equation (11) gives the classical Lapicque-Blair-Hill [11-13] equation for the strengthduration curve,

$$
I_{s}=\frac{I_{\mathrm{rh}}}{1-e^{-t_{s} / \tau}}
$$

with rheobase

$$
I_{\mathrm{rh}}=\frac{\phi_{1}(0)}{\lambda_{1} \int_{0}^{\infty} \phi_{1}(x) u_{*}(x) \mathrm{d} x}=\frac{45}{64} \pi \theta^{3 / 2}
$$

and chronaxie

$$
\tau=\left(\lambda_{1}\right)^{-1}=\frac{4}{5 \theta} .
$$

Fig. 2 illustrates the quality of the analytical critical curves $(13)$ and $(14,15,16)$, both compared to the curves obtained by direct numerical simulations for the quadratic nonlinearity (12) valid for small $\theta$, and the original cubic nonlinearity (2). For the chosen parameter values, the error introduced by linear approximation of the stable manifold of the critical nucleus is of the same order of magnitude as the error introduced by the quadratic approximation of the nonlinearity.

In conclusion, we have obtained analytical expressions for initiation criteria for a concrete simple example. Such criteria were obtained experimentally and numerically and any analytical expression was through fitting; we have deduced it mathematically $a b$ initio, via a clearly defined procedure. The expressions are simple enough to be useful in practice, but the procedure of obtaining them is probably more important as it can be extended to other models. The expression for the strength-extent 


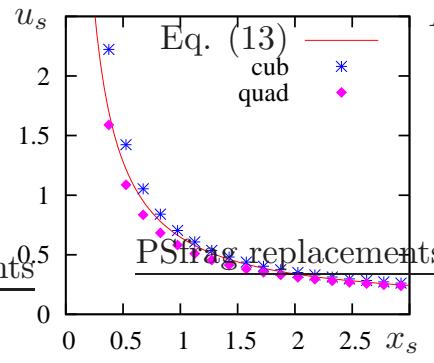

(a)

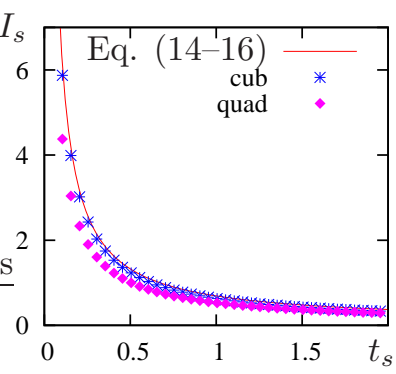

(b)
FIG. 2: (color online) Comparison of analytical predictions with numerical simulations. (a) Strength-extent curves for rectangular initial conditions. (b) Strength-duration curves for point stimulation. Red solid lines: analytical approximations, (13) for (a) and $(14,15,16)$ for (b). Blue stars ("cub"): numerical results for cubic kinetics (2). Magenta diamonds ("quad"): numerical results for quadratic kinetics (12).

curve is specific for the ZFK equation and will have a different form for a different model. However, the temporal strength-duration curve is universal, up to the values of two constants, and it coincides precisely with a classical form used for over 100 years for analytical fitting of empirical data.

The general principle, linear approximation of the (center-)stable manifold of the critical solution, easily admits extensions, e.g. for different temporal and spatial profiles of the initiation stimuli, different initiation protocols, possibility of optimization, say with respect to the total energy required to initiate a wave etc.

It also can be extended to other threshold systems, whenever the critical solution can be identified, including those having critical solutions which are not critical nuclei [22]. In such systems, an additional problem is anticipated, as one cannot use the even $(x \rightarrow-x)$ embedding and have to take into account the translational symmetry of the problem posed on the whole real axis.

Authors are grateful to J. Brindley for inspiring discussions and R. Suárez Antola for bibliographic advice. The study has been supported in part by EPSRC grant GR/S75314/01 and MacArthur Foundation grant 7135601 .

[1] A. V. Gurevich and R. G. Mints, Rev. Mod. Phys. 59, 941 (1987).
[2] M. C. Cross and P. C. Hohenberg, Rev. Mod. Phys. 65, 851 (1993).

[3] J. P. Keener and J. Sneyd, Mathematical Physiology (Springer, 1998).

[4] A. G. Merzhanov and E. N. Rumanov, Rev. Mod. Phys. 71, 1173 (1999).

[5] V. V. Bychkov and M. A. Liberman, Physics Reports 325, 115 (2000).

[6] J. D. Murray, Mathematical Biology: I. An Introduction (Springer, 2002).

[7] V. Krinsky and H. Swinney, eds., Wave and patterns in biological and chemical excitable media (North-Holland, Amsterdam, 1991).

[8] A. V. Holden, M. Markus, and H. G. Othmer, eds., Nonlinear wave processes in excitable media (Plenum Press, 1991).

[9] D. P. Zipes and J. Jalife, eds., Cardiac electrophysiology: From cell to bedside (W B Saunders Co, 2004).

[10] A. A. Shah, J. Brindley, A. C. McIntosh, and J. Rademacher, Nonlinear Analysis: Real World Applications 9, 562 (2008).

[11] L. Lapicque, J Physiol (Paris) (1907).

[12] H. A. Blair, J Gen Physiol 15, 709 (1932).

[13] A. V. Hill, Proc R Soc Lond (Biol) 119, 305 (1936).

[14] W. Rushton, Proc. Roy. Soc. Lond. ser. B 124, 210 (1937).

[15] D. Noble, J. Physiol. 226, 573 (1972).

[16] R. E. Suarez Antola and A. C. Sicardi Schifino, Physica D 89, 427 (1996).

[17] J. C. Neu, R. S. Preissig, and W. Krassowska, Physica D 102, 285 (1997).

[18] H. P. McKean and V. Moll, Bull. AMS 12, 255 (1985).

[19] G. Flores, J. Diff. Eq. 80, 306 (1989).

[20] G. Flores, SIAM J. Math. Anal. 22, 392 (1991).

[21] V. Moll and S. I. Rosencrans, SIAM J. Appl. Math. 50, 1419 (1990).

[22] I. Idris and V. N. Biktashev, Phys. Rev. E 76, 021906 (2007).

[23] Y. B. Zel'dovich and D. A. Frank-Kamenetsky, Doklady AN SSSR 19, 693 (1938).

[24] R. FitzHugh, Biophys. J. 1, 445 (1961).

[25] J. Nagumo, S. Arimoto, and S. Yoshizawa, Proc. IRE 50, 2061 (1962).

[26] I. S. Gradshteyn and I. M. Rhyzhik, Tables of integrals, series, and products (Academic Press, 2000), sixth ed.

[27] It is a stable manifold in (3) and in the even-function subspace of (6), and a center-stable manifold in the full phase space of (6). 